\title{
Electron affinities of organic materials used for organic light-emitting diodes: A low-energy inverse photoemission study
}

\author{
${\text { Hiroyuki Yoshida }{ }^{*} \text {, Kei Yoshizaki² }}^{2}$ \\ ${ }^{1}$ Institute for Chemical Research, Kyoto University, Uji, Kyoto 611-0011, Japan \\ 2 Idemitsu Kosan Co. Ltd., 1280 Kami-izumi, Sodegaura, Chiba, 299-0293, Japan
}

\begin{abstract}
The electron affinity (EA) of an organic semiconductor is a measure of the electron transport level. Although reliable values of the EA are required for designing the device architecture of organic light-emitting diodes (OLED), there were no appropriate methods. Recently we have developed low-energy inverse photoemission spectroscopy which enables us to determine the EA of organic materials in solid with the precision required for research of OLED. Using this new technique, we precisely determined EA of typical OLED materials, TCTA, CBP, Ir(ppy) $)_{3}$, BCP, Alq3 and Liq as well as a newly developed dopant $4 \mathrm{CzIPN}$. The obtained electron affinities are generally smaller by about $1 \mathrm{eV}$ than the commonly believed values urging the reconsideration of the electron injection/ transport mechanisms in OLED. We also compare EAs determined by various experimental and calculation methods for 29 materials. The results show that the reduction potential gives a reasonable estimate rather than the optical gap and ionization energy.
\end{abstract}

Keywords: low-energy inverse photoemission spectroscopy; electron affinity; LUMO level; organic light-emitting diode;

*corresponding author: Hiroyuki Yoshida

TEL: +81-774-38-3083

FAX: +81-774-38-3084

Email: yoshida@e.kuicr.kyoto-u.ac.jp 


\section{Introduction}

Organic light-emitting diodes (OLED) are a promising new technology for flat-panel displays and interior lighting. Since the first report of the electroluminescence in organic material in 1960s [1], external quantum efficiency and operating voltage are markedly improved by harvesting the triplet exciton [2-7], controlling the molecular orientation of emitter molecule [7-9] and introducing multilayer structures [10-13]. Particularly, multilayer devices can decrease the carrier injection barriers at the interfaces and efficiently confine the carriers into the emissive layer. Following the monumental development of the two layer structure by Tang which lowered the operating voltage to $10 \mathrm{~V}$ and achieved the brightness of $1000 \mathrm{~cd} \mathrm{~cm}^{-2}$ [10], doping emitting material in host [11], insertion of exciton and hole block layer [12], and electron transport layer [13] has been demonstrated. Now as many as 5 or 7 layers are common.

Designing an efficient multilayer structure requires knowing the precise energy of the hole and electron transport levels of constituent materials [12, 14], corresponding to the highest occupied molecular orbital-derived levels (HOMO levels) and the lowest unoccupied molecular orbital-derived levels (LUMO levels), respectively. While the HOMO levels have been examined extensively using photoemission spectroscopy (PES) and photoemission yield spectroscopy (PYS), there was no appropriate method available to accurately measure the LUMO levels. While the reduction potentials measured using cyclic-voltammetry in solution are often used to estimate the electron affinity (the bottom of the LUMO level with respect to the vacuum level), the value measured in solution is inherently different from the electron affinity of solid materials. The electron affinity is also frequently estimated by adding the optical gap and the ionization energy (the top of the HOMO level with respect to the vacuum level). The optical gap is usually smaller than the transport gap by an amount of $0.2-1.0 \mathrm{eV}$ which is interpreted as the exciton binding energy [15-17], causing the electron affinity to be overestimated.

In principle, inverse photoemission spectroscopy (IPES) can give the electron affinity relevant to the electron transport in the solid material[16, 18]. Actually the electron affinity determined by IPES is often regarded as the reference value[19, 20]. In this technique, an electron is introduced to the surface of sample material and a photon is emitted due to the radiative transition the unoccupied levels. From the onset of the photon signal, the electron affinity is determined. The uncertainty

involved is, however, often assumed to $\pm 0.35 \mathrm{eV}$ [19], substantially higher than PES, its 
complementary method for determining the ionization energy. The fundamental problem originates from the low cross section of IPES process which is five orders of magnitude smaller than PES [21]. A high intensity electron beam is required to gain sufficient signal intensity resulting in serious damage to organic samples [22, 23], and the weak photon signal have to be detected using specially designed photon detector with a high sensitivity and an inadequate energy resolution [24].

Recently, we developed low-energy inverse photoemission spectroscopy (LEIPS) which solves the both issues [25, 26]. The kinetic energy of electron is lowered below $5 \mathrm{eV}$, the damage threshold of most organic materials [27]. By decreasing the electron energy, the photons in the near ultraviolet range emit which can be analyzed using a high-resolution bandpass filter. So far we have applied LEIPS to various organic materials [26, 28-32] demonstrating that the electron affinities are determined with the precision similar to PES without damaging the organic samples.

In this study, we determine the electron affinities of organic materials relevant to OLEDs, including a hole transport material, tris(4-carbazoyl-9-ylphenyl)amine (TCTA), a host material, 4,4'-bis(carbazol-9-yl)biphenyl (CBP) [3], a phosphorescent material, tris(2-phenylpyridinato)iridium(III) $\quad\left(\operatorname{Ir}(\mathrm{ppy})_{3}\right) \quad$ [3], electron transport materials, (2,9-dimethyl-4,7-diphenyl-1,10-phenanthroline) (BCP) [3, 33], 8-hydroxyquinolinolato-lithium (Liq) [34] and tris(8-hydroxyquinolinato)aluminium (Alqu ${ }_{3}$ [10], a hole injection material, 2,3,6,7,10,11-hexacyano-1,4,5,8,9,12-hexaazatriphenylene (HAT-CN) and a recently developed $\begin{array}{llll}\text { thermally activated } & \text { delayed-fluorescent }\end{array}$ 1,2,3,5-tetrakis(carbazol-9-yl)-4,6-dicyanobenzene (4CzIPN) [6]. In addition to these most frequently used materials, we determine electron affinities of 22 materials designed and developed specially for the OLED application by Idemitsu Kosan Co.,LTD. The values determined by LEIPS are compared with those obtained by other commonly used experimental methods, such as the reduction potential and the sum of ionization energy and optical gap as well as calculated values of the LUMO orbital energy, the electron affinity, and the sum of the ionization energy and singlet excitation energy based on the density functional method.

\section{Experimental}

The thin films were prepared by vacuum deposition method on a 100-nm-thick indium-tin oxide 
(ITO) film or an 80-nm-thick $\mathrm{Al}$ film on a glass plate at a pressure lower than $10^{-5} \mathrm{~Pa}$. Prior to the vacuum deposition, the ITO coated glass was treated by the UV ozone followed by Ar plasma. The thicknesses of the films were 5,25 and $50 \mathrm{~nm}$ while the deposition rate was kept to $0.1 \mathrm{~nm} \mathrm{~s}{ }^{-1}$ monitored by a quartz micro balance.

The prepared films were exposed to air and introduced to the LEIPS apparatus evacuated to lower than $10^{-7} \mathrm{~Pa}$. The detail of the experimental apparatus is described elsewhere [35]. Mono-energetic electron beam was introduced to the sample surface with the sample current between 0.4 and $0.5 \mu \mathrm{A}$. Under this condition, any spectral change due to the sample degradation was not observed. The emitted photons are collected and focused using a quartz lens into the photon detector consisting of a bandpass filter and photomultiplier tube. The bandpass filters with the following nominal center wavelengths (the corresponding photon energies in the parenthesis) were used: $254 \mathrm{~nm}(4.885 \mathrm{eV}), 260 \mathrm{~nm}(4.785 \mathrm{eV}), 280 \mathrm{~nm}(4.455 \mathrm{eV}), 285 \mathrm{~nm}(4.377 \mathrm{eV}), 335 \mathrm{~nm}(3.713$ $\mathrm{eV}), 387 \mathrm{~nm}(3.203 \mathrm{eV}), 434 \mathrm{~nm}(2.859 \mathrm{eV})$. Overall energy resolution was about $0.3 \mathrm{eV}$.

The ionization energies were measured using PYS in air on Riken Keiki AC-3 for the 50-nm-thick films on the ITO glass at the light intensity of $1 \mathrm{nW}$. The square root of photoemission yield is plotted against the photon energy and the ionization energy is determined as the onset of the spectrum. The optical gaps were measured in toluene solution with the concentration of about $10^{-5}$ mol $\mathrm{L}^{-1}$ on Hitachi UV-vis spectrometer U3310. Cyclic voltammetry were performed using a potentiostat (ALS Co., Ltd, Model 2325). The sample was dissolved in N,N-dimethylformamide (DMF) and tetrabutylammonium perchlorate $\left(\mathrm{t}-\mathrm{Bu}_{4} \mathrm{NClO}_{4}\right)$ was mixed as a supporting electrolyte. Glass carbon, $\mathrm{Pt}$, and $\mathrm{Ag} / \mathrm{AgCl}$ were used as the working electrode, the counter electrode and the reference electrode, respectively. The first oxidation potential of ferrocene was used as a reference.

\section{Calculation}

The density functional (DFT) calculations were performed for a single molecule using the hybrid density functional B3LYP with the 6-31G(d) basis set on Gaussian 09 program package [36]. The adiabatic electron affinity was obtained as the energy difference between the anion and neutral molecule at the optimized geometry for each state. The singlet excitation energy is calculated with time-dependent DFT (TD-DFT) at the same level. 


\section{Results and Discussion}

The aim of this work is to determine the electron affinities of organic materials as a measure of electron transport levels in OLED. This means that the obtained values should be specific to the material and not depend on film thickness or substrate. Thus we first pursue the optimal condition for the film thickness and substrates taking BCP as an example. Figure 1a shows the thickness dependence of LEIPS spectra for BCP films on ITO. We observed no apparent features from the substrate ITO or effects of sample charging in between 5 and $20 \mathrm{~nm}$. Beyond $50 \mathrm{~nm}$, the spectral feature becomes unclear and shifts toward the low-energy side due to the sample charging. We therefore use 5-nm thick films throughout this work. Next, BCP is deposited on the substrates of ITO and Al to compare the spectra in Figure 1b. The onset energies are similar, while the background signal increases in the higher energy region of the BCP film on $\mathrm{Al}$. The background photon signals are likely generated due to the inelastically scattered electrons [37], which is consistent with the finding that the features around the onset are similar in the both spectra. The electron affinity is thus able to be determined for either of the substrates. In this work, ITO is used because the spectral features are more clearly observed. In order to examine the effect of the ambient air, a $5 \mathrm{~nm}$-thick BCP film on ITO was prepared and measured without exposing to air (in-situ). As shown in Figure 1c, the spectra show no discernible difference in the onset energy or spectral line shape and confirm that the electron affinities are unaffected. The result is consistent with previous photoemission results where air exposure resulted in a shift of the Fermi level while the ionization energy was left unchanged [38]. 
(a)

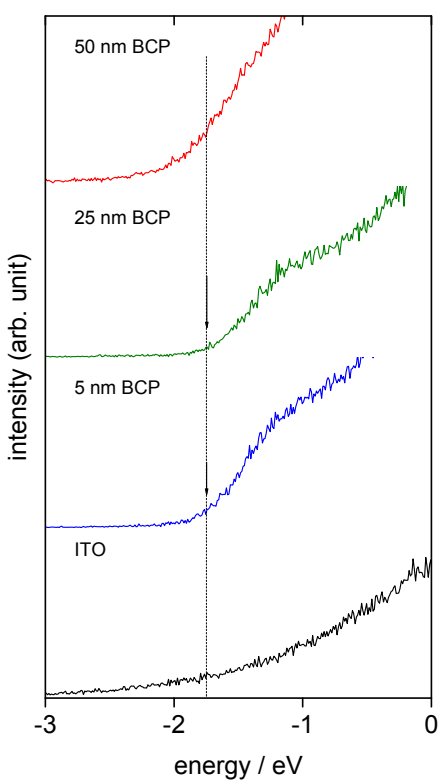

(b)

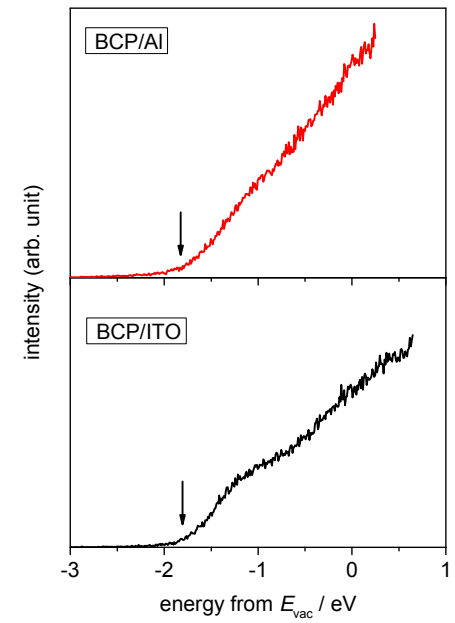

(c)

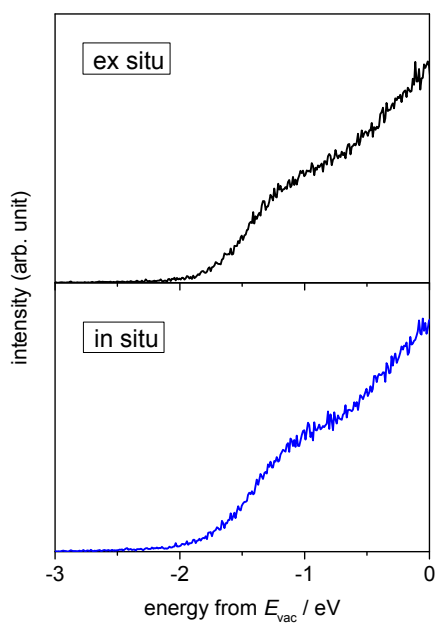

Figure 1: LEIPS spectra taken at $335 \mathrm{~nm}$ for BCP films. (a) Spectra of BCP/ITO with film thickness between 0 and $50 \mathrm{~nm}$. (b) The effect of the substrates, $\mathrm{Al}$ and ITO with the film thickness of $5 \mathrm{~nm}$. (c) Spectra of 5־nm-thick films on ITO with (ex-situ) and without (in-situ) exposed to air.

Based on the BCP results, we measured the LEIPS spectra of TCTA, CBP, Ir(ppy) 3 , $4 \mathrm{CzIPN}, \mathrm{BCP}$, Liq, Alq3 and HAT-CN for 5-nm-thick films on ITO substrates. The results together with the molecular structures are shown in Figure 2. The measurements were made at three different detection wavelengths to reduce the systematic error [25]. The onset energies with respect to the vacuum levels which were determined by the onset of the sample current, we determined the electron affinities as listed in Table 1. Only the onset of HAT-CN was not observed using any available bandpass filters indicating that the electron affinity of HA- $\mathrm{CN}$ is larger than $4.9 \mathrm{eV}$. The uncertainty is estimated statistically from multiple measurements. In addition to the precisions shown in Table 1, there is ambiguity of less than $0.1 \mathrm{eV}$ in determining the vacuum levels and the onset of LUMO level. The electron affinity is also affected by the crystallinity [28] and molecular orientation [39] of the sample film, which depend on the film preparation conditions. In this work, the sample films were prepared under as close condition as the OLED device fabrication so that the determined energy parameters represent the energy levels relevant to the OLED performance. 
In Table 1 , the electron affinities $A$ by LEIPS, and the electron affinities estimated by other methods are compiled together with the ionization energies $I$, the optical gaps $E_{\mathrm{G}}$ opt, and the reduction potentials $E_{\text {red. }}$ The ionization energies are in a good agreement with the reported values (TCTA[40], CBP[41], BCP[42], and Alq [43]). The electron affinity by LEIPS is close to that obtained by the conventional IPES for CBP (1.9 eV) [41], while different for TCTA (2.14 eV) [40] which is most likely affected by the sample damage demonstrating the advantage of LEIPS.

Table 1: The electron affinity determined by LEIPS $A$, the ionization energy by PYS $I$, the optical gap $E_{\mathrm{G}}$ opt, the electron affinities obtained by the ionization energy and optical gap $A_{\mathrm{i}+\mathrm{opt}}$, and the reduction potential $E_{\text {red. }}$

\begin{tabular}{|c|c|c|c|c|c|}
\hline & $A / \mathrm{eV}$ & $I / \mathrm{eV}$ & $E_{\mathrm{G}}$ opt $/ \mathrm{eV}$ & $A_{\mathrm{i}+\mathrm{opt}} / \mathrm{eV}$ & $E_{\text {red }} / \mathrm{eV}$ \\
\hline TCTA & $1.59 \pm 0.09$ & 5.80 & 3.26 & 2.54 & - \\
\hline $\mathrm{CBP}$ & $1.75 \pm 0.05$ & 6.08 & 3.39 & 2.69 & $2.75^{\mathrm{a}}$ \\
\hline Liq & $1.85 \pm 0.06$ & 5.69 & 2.76 & 2.93 & $3.15^{\mathrm{b}}$ \\
\hline $\operatorname{Ir}(\mathrm{ppy})_{3}$ & $1.86 \pm 0.05$ & 5.27 & 2.38 & 2.89 & 2.71 \\
\hline $\mathrm{BCP}$ & $1.89 \pm 0.04$ & 6.47 & 3.35 & 3.12 & $2.53^{\mathrm{a}}$ \\
\hline $\mathrm{Alq}_{3}$ & $2.06 \pm 0.03$ & 5.80 & 2.53 & 3.27 & $2.30^{\mathrm{c}}$ \\
\hline 4CzIPN & $2.81 \pm 0.05$ & 5.91 & 2.61 & 3.44 & 1.68 \\
\hline HAT-CN & $>4.9$ & - & - & - & 0.97 \\
\hline
\end{tabular}

aRef. [19]; bRef. [34] measured in acetonitrile; cRef. [44]. 

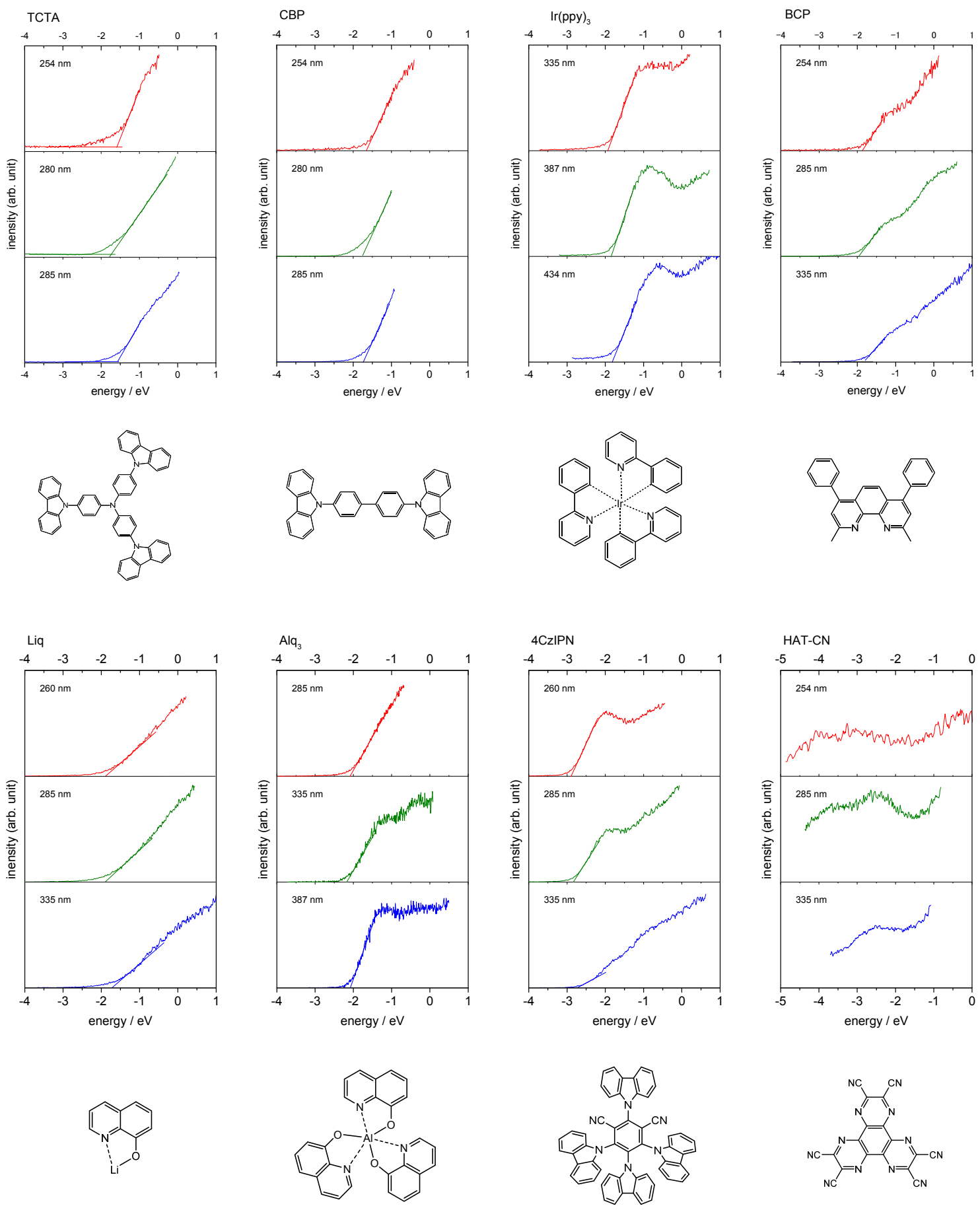

Figure 2: Low-energy inverse photoemission (LEIPS) spectra of materials frequently used for OLED together with the molecular structures. The spectra are measured at three different wavelengths which are indicated in the panels. The abscissa is the energy with respect to the vacuum level. 
In the literature of OLED, the following electron affinities, normally obtained by the ionization energies and optical gaps, are often cited: $2.8 \mathrm{eV}$ for CBP, $3.2 \mathrm{eV}$ for $\mathrm{BCP}$ [3, 12], $2.7 \mathrm{eV}$ for Alq3 [3], $2.2 \mathrm{eV}$ for TCTA [14], $2.7 \mathrm{eV}$ for $\operatorname{Ir}(\mathrm{ppy})$ [14], which are in good agreements with $A_{\text {i+opt }}$ in Table 1. These electron affinities are about $1 \mathrm{eV}$ higher (i.e. the LUMO levels is deeper) than those determined in this work. Such large differences require the design strategy and device physics of OLED based on the earlier values to be reconsidered.

The exciton binding energies are evaluated from the difference between the electron affinities $A$ and that deduced from the ionization energy and optical gap $A_{\mathrm{i}+\mathrm{opt}}=I-E_{\mathrm{G}} \mathrm{opt}, 0.96 \mathrm{eV}$ for TCTA, $0.94 \mathrm{eV}$ for $\mathrm{CBP}, 1.03 \mathrm{eV}$ for $\mathrm{Ir}(\mathrm{ppy})_{3}, 1.24 \mathrm{eV}$ for $\mathrm{BCP}$ and $1.21 \mathrm{eV}$ for Alq3. These values are substantially larger than $0.5 \mathrm{eV}$ of those for the fullerenes $\left(\mathrm{C}_{60}\right.$ and $\left.\mathrm{PC}_{61} \mathrm{BM}\right)$ [29] and pentacene [31].

In order to make further comparisons between the electron affinities determined by LEIPS and those estimated from other experimental and theoretical methods for a broad range of materials, we have examined the energy parameters of additional 22 compounds developed for OLED. The LEIPS measurements were carried out at the wavelength of $285 \mathrm{~nm}$ for the 5 -nm-thick films. The resultant electron affinities by LEIPS $A$ are compared with $A_{\text {i+opt }}$ and $E_{\text {red }}$ in Figures $3 \mathrm{a}$ and $3 b$, respectively.

The relation between $A$ and $A_{\mathrm{i}+\mathrm{opt}}$ scatters and shows poor agreement. A linear regression analysis to the data gives a slope of $0.59 \pm 0.12$ and a intercept $1.61 \pm 0.25 \mathrm{eV}$ with a correlation coefficients of 0.4671. The difference in energy between $A$ and $A_{\mathrm{i}+\text { opt }}$ can be interpreted as the exciton binding energy. The result suggests the exciton binding energies is on the order of $1 \mathrm{eV}$ and vary strongly depended on the materials. Although $A_{\mathrm{i}+\mathrm{opt}}$ is frequently used in place of $A, A_{\mathrm{i}+\text { opt }}$ should not be scaled, at least, for a reasonable estimation of $A$.

On the other hand, the relation between the electron affinity $A$ and the reduction potential $E_{\text {red }}$ are in a good agreement as shown in Figure 3b. A linear fit to the data gives a slope of $0.90 \pm 0.08$ and a intercept of $-4.30 \pm 0.17 \mathrm{eV}$ with a correlation coefficients of 0.8958 . The reduction potentials have been compared with the electron affinities in the gas $[45,46]$ and solid phases [19] in the 
literature. From these values, the following relation yields for transforming $E_{\text {red }}$ to the electron affinity $A_{\text {red, }}$

$$
A_{\text {red }}=1.112 E_{\text {red }}+4.78
$$

The slope of $1.112 \mathrm{eV}$ is in a good agreement with the previous report [19] while the constant is close to the first oxidation potential of ferrocene $(4.8 \mathrm{eV})$ used as the reference electrode. Note that the slope and constant in Equation 1 may depend on such experimental conditions as the solvent, electrodes, and supporting electrolyte. One should calibrate the parameters before use.

(a)

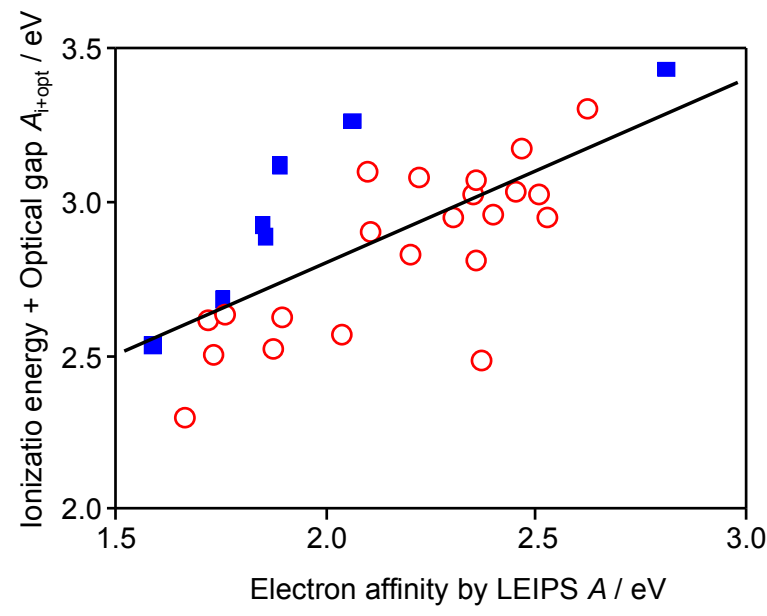

(b)

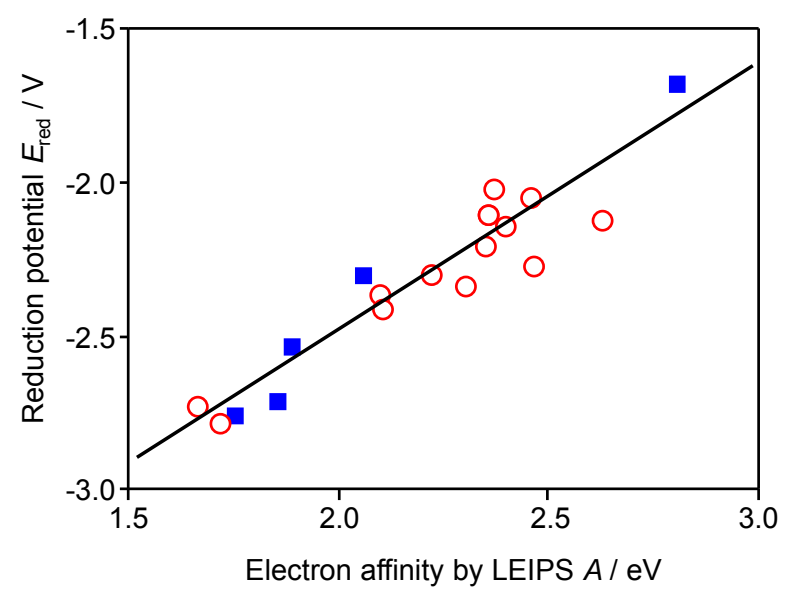

Figure 3: The electron affinity determined by LEIPS is compared with other experimental methods: (a) the sum of ionization energy and optical gap and (b) the reduction potential. The compounds listed in Table 1 (filled square), and the other materials (open circle). A linear fit to all the data is shown by a solid line in each panel.

According to the recent development of computational resources, the electronic properties of organic materials are easily estimated using density functional (DFT) calculations on a single molecule. We calculate the electron affinities by three methods: (1) the Kohn-Sham LUMO energy, (2) the electron affinity calculated as difference in total energy between the anionic $E$ - and neutral $E_{0}$ molecule, (3) sum of the Kohn-Sham HOMO orbital energy and singlet excitation energy. 
(a)

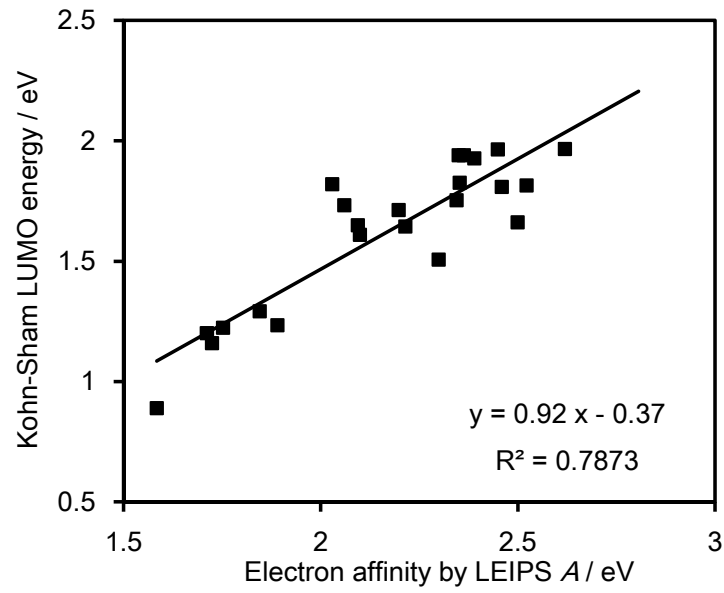

(c)

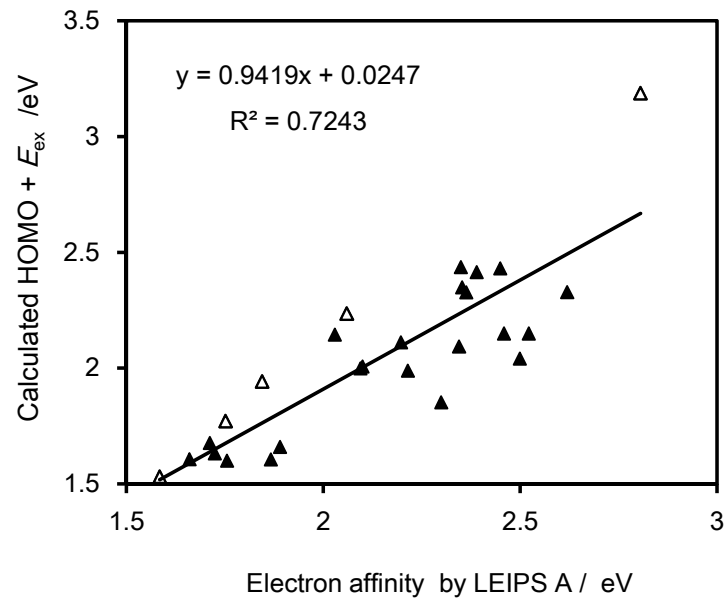

(b)

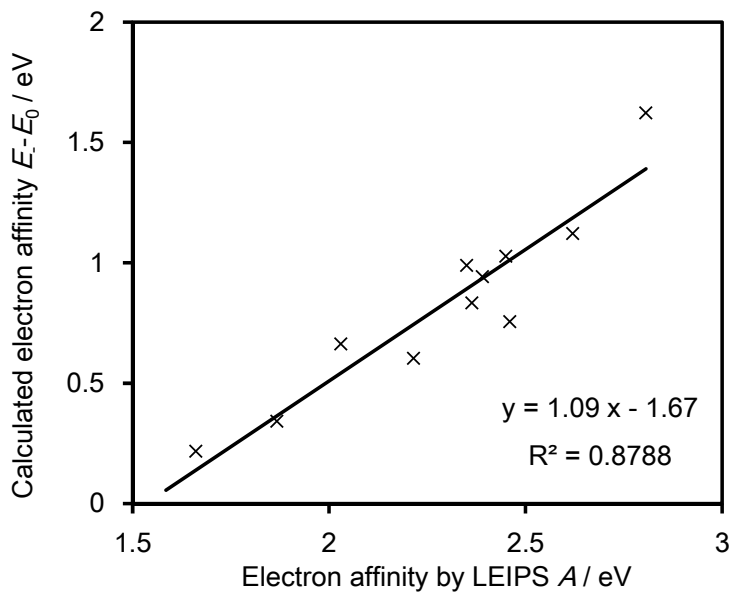

(d)

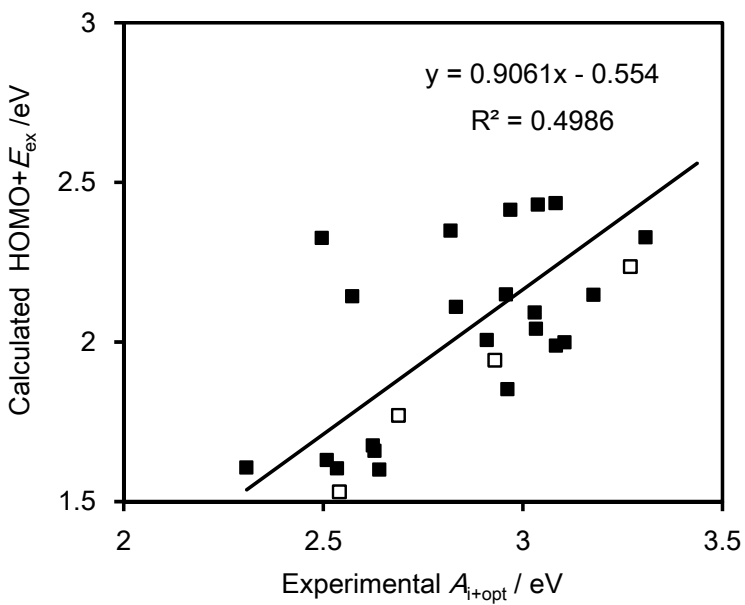

Figure 4: The calculated electron affinities compared with the experimental data. (a) The Kohn-Sham LUMO energy and (b) the adiabatic electron affinity as difference in total energy between the anion $E$ - and neutral molecule $E_{0}$ are plotted against $A$ determined by LEIPS. The sum of the Kohn-Sham HOMO energy and the singlet excitation energy $E_{\text {ex }}$ is compared with (c) $A$ by LEIPS and (d) the difference between the experimental ionization energy and optical gap $A_{\text {itopt. }}$.

At first, the calculated values by (1)-(3) are compared with the electron affinities determined by LEIPS in the panels (a)-(c) of Figure 4, respectively. The calculated LUMO energies (1) show strong correlation with experimental $A$ with a correlation coefficient of 0.7873 . The calculated electron affinities (2) show slightly better agreement with a correlation coefficient of 0.8788 . In view of the higher calculation cost for the method (2), the simple Kohn-Sham orbital energy seems to be sufficient. These findings are essentially the same as the earlier report[20]. In any case, a proper scaling is necessary to estimate the experimental $A$. 
On the other hand, the calculated electron affinity deduced from the HOMO orbital energy and the singlet excitation energy (3) shows fairly good correlation with experimental $A$ by LEIPS with a coefficient of 0.7243 , meaning that the optical gap can be evaluated from the UV-vis spectrum measured in solution. Nevertheless, the agreement between the experimental $A$ and $A_{\mathrm{i}+\text { opt }}$ is poor. In order to clarify the reason, the calculated value (3) is compared with the experimental ionization energy and the optical gap $A_{\mathrm{i}+\mathrm{opt}}$ in Figure $4 \mathrm{~d}$. The agreement is unsatisfactory with the correlation factor of 0.4986. The finding suggests that the optical gaps were not determined satisfactorily in the practical experiment partially because the onset energy was not always clear in the spectra. This ambiguity may be the main reason of the disagreement demonstrated in Figure 3a though it should be merely a technical and not fundamental problem.

\section{Conclusion}

The LUMO levels of 30 organic semiconductors used for OLED were examined using low-energy inverse photoemission spectroscopy (LEIPS). The materials includes the commonly used molecules, TCTA, CBP, Ir(ppy) 3 , BCP, Liq, Alq 3 , HAT-CN as well as the newly developed thermally activated delayed fluorescence material, 4 CzIPN. The electron affinities of 29 materials are determined with the uncertainty similar to PES or PYS, while that of HAT-CN lay outside of the maximum measurement range of $4.9 \mathrm{eV}$.

The electron affinities in this work are systematically smaller (the LUMO levels are higher) than the widely accepted values (e.g. Refs $[3,12,14]$ ) due to the large exciton binding energy of about1 eV. This large difference suggests the energy level alignment between the multi-layers in OLED, particularly, of electron injection from cathode to organic layer should be reconsidered. The workfunctions of typical cathode materials such as $\mathrm{Al}, \mathrm{Ag}, \mathrm{Mg}$ are around $4 \mathrm{eV}$ while the electron affinities of materials studied in this work is in the range between 1.6 and $2.8 \mathrm{eV}$. This implies that the electron injection barrier exceeds $1 \mathrm{eV}$ even when the vacuum level shift as much as $1 \mathrm{eV}$ [47, 48] is taken into account.

The electron affinities obtained by LEIPS are compared with those obtained by other experimental and theoretical methods. We found the electron affinities determined by LEIPS correlated well with those derived from the reduction potential in solution, the Kohn-Sham LUMO energy, and the 
electron affinity as difference between the anion and neutral molecules by DFT calculation. This means that the electron affinity of single molecule provides a reasonable estimation of that in organic solid for the materials studied in this work. For example, the reduction potential converted by Equation 1 gives a reasonable estimate for the electron affinity. In contrast, the electron affinities estimated based on the optical gap show poor agreement with the values determined by LEIPS. In OLEDs, amorphous films are preferentially used where the intermolecular interactions are averaged. Further the orientation dependence of the ionization energy should play a limited role [39, 49]. Though good agreements between the electron affinities determined by LEIPS and those based on the single molecules are observed, the relation expressed by Equation 1 should be restricted to the OLED materials and may not be generalized.

\section{Acknowledgements}

This work is supported by JST PRESTO. The authors thank Dr. Richard Murdey for proofreading. 


\section{References}

[1] M. Pope, P. Magnante, H.P. Kallmann, J. Chem. Phys., 38 (1963) 2042.

[2] M.A. Baldo, D.F. O'Brien, Y. You, A. Shoustikov, S. Sibley, M.E. Thompson, S.R. Forrest, Nature, 395 (1998) 151-154.

[3] M.A. Baldo, S. Lamansky, P.E. Burrows, M.E. Thompson, S.R. Forrest, Appl. Phys. Lett., 75 (1999) 4-6.

[4] C. Adachi, M.A. Baldo, M.E. Thompson, S.R. Forrest, J. Appl. Phys., 90 (2001) 5048-5051.

[5] D.Y. Kondakov, T.D. Pawlik, T.K. Hatwar, J.P. Spindler, J. Appl. Phys., 106 (2009).

[6] H. Uoyama, K. Goushi, K. Shizu, H. Nomura, C. Adachi, Nature, 492 (2012) 234-+.

[7] H. Kuma, C. Hosokawa, Science and Technology of Advanced Materials, 15 (2014).

[8] D. Yokoyama, A. Sakaguchi, M. Suzuki, C. Adachi, Org. Electron., 10 (2009) 127-137.

[9] D. Yokoyama, Y. Setoguchi, A. Sakaguchi, M. Suzuki, C. Adachi, Adv. Func. Mater., 20 (2010) 386-391.

[10] C.W. Tang, S.A. Vanslyke, Appl. Phys. Lett., 51 (1987) 913-915.

[11] C.W. Tang, S.A. Vanslyke, C.H. Chen, J. Appl. Phys., 65 (1989) 3610-3616.

[12] V.I. Adamovich, S.R. Cordero, P.I. Djurovich, A. Tamayo, M.E. Thompson, B.W. D'Andrade, S.R.

Forrest, Org. Electron., 4 (2003) 77-87.

[13] A.P. Kulkarni, C.J. Tonzola, A. Babel, S.A. Jenekhe, Chem. Mater., 16 (2004) 4556-4573.

[14] M. Mesta, M. Carvelli, R.J. de Vries, H. van Eersel, J.J.M. van der Holst, M. Schober, M. Furno, B.

Luessem, K. Leo, P. Loebl, R. Coehoorn, P.A. Bobbert, Nature Mater., 12 (2013) 652-658.

[15] J.L. Bredas, J. Cornil, A.J. Heeger, Adv. Mater., 8 (1996) 447-452.

[16] I.G. Hill, A. Kahn, Z.G. Soos, R.A. Pascal, Chem. Phys. Lett., 327 (2000) 181-188.

[17] M. Knupfer, Appl. Phys. A-Mater. Sci. Process., 77 (2003) 623-626.

[18] S. Krause, M.B. Casu, A. Scholl, E. Umbach, New J. Phys., 10 (2008) 085001.

[19] P.I. Djurovich, E.I. Mayo, S.R. Forrest, M.E. Thompson, Org. Electron., 10 (2009) 515-520.

[20] P.E. Schwenn, P.L. Burn, B.J. Powell, Org. Electron., 12 (2011) 394-403.

[21] P.D. Johnson, S.L. Hulbert, Rev. Sci. Instrum., 61 (1990) 2277-2288.

[22] J. Tsutsumi, D. Yamamoto, H. Yoshida, N. Sato, Synth. Met., 158 (2008) 934-938.

[23] Z. Li, S.Q. Sun, X. Li, R. Schlaf, Appl. Phys. Lett., 104 (2014) 021606.

[24] D.R.T. Zahn, G.N. Gavrila, M. Gorgoi, Chem. Phys., 325 (2006) 99-112.

[25] H. Yoshida, Chem. Phys. Lett., 539-540 (2012) 180-185.

[26] H. Yoshida, Anal. Bioanal. Chem., 406 (2014) 2231-2237.

[27] B. Boudaiffa, P. Cloutier, D. Hunting, M.A. Huels, L. Sanche, Science, 287 (2000) 1658-1660.

[28] Y. Zhong, S. Izawa, K. Hashimoto, K. Tajima, T. Koganezawa, H. Yoshida, J. Phys. Chem. C, 119 (2015) 23-28.

[29] H. Yoshida, J. Phys. Chem. C, 118 (2014) 24377-24382.

[30] Y. Ie, M. Karakawa, S. Jinnai, H. Yoshida, A. Saeki, S. Seki, S. Yamamoto, H. Ohkita, Y. Aso, Chem. Comm., 50 (2014) 4123-4125. 
[31] W. Han, H. Yoshida, N. Ueno, S. Kera, Appl. Phys. Lett., 103 (2013) 123303.

[32] S. Fabiano, H. Yoshida, Z.H. Chen, A. Facchetti, M.A. Loi, ACS Appl. Mater. Interfaces, 5 (2013) 4417-4422.

[33] Y. Kijima, N. Asai, S. Tamura, Jpn. J. Appl. Phys. Part 1 - Regul. Pap. Short Notes Rev. Pap., 38 (1999) 5274-5277.

[34] C. Schmitz, H.W. Schmidt, M. Thelakkat, Chemistry of Materials, 12 (2000) 3012-3019.

[35] H. Yoshida, Rev. Sci. Instrum., 85 (2014) 016101.

[36] M. J. Frisch, G. W. Trucks, H. B. Schlegel, G. E. Scuseria, M. A. Robb, J. R. Cheeseman, G. Scalmani, V. Barone, B. Mennucci, G. A. Petersson, H. Nakatsuji, M. Caricato, X. Li, H. P. Hratchian, A. F. Izmaylov, J. Bloino, G. Zheng, J. L. Sonnenberg, M. Hada, M. Ehara, K. Toyota, R. Fukuda, J. Hasegawa, M. Ishida, T. Nakajima, Y. Honda, O. Kitao, H. Nakai, T. Vreven, J. A. Montgomery, Jr., J. E. Peralta, F. Ogliaro, M. Bearpark, J. J. Heyd, E. Brothers, K. N. Kudin, V. N. Staroverov, T. Keith, R. Kobayashi, J. Normand, K. Raghavachari, A. Rendell, J. C. Burant, S. S. Iyengar, J. Tomasi, M. Cossi, N. Rega, J. M. Millam, M. Klene, J. E. Knox, J. B. Cross, V. Bakken, C. Adamo, J. Jaramillo, R. Gomperts, R. E. Stratmann, O. Yazyev, A. J. Austin, R. Cammi, C. Pomelli, J. W. Ochterski, R. L. Martin, K. Morokuma, V. G. Zakrzewski, G. A. Voth, P. Salvador, J. J. Dannenberg, S. Dapprich, A. D. Daniels, O. Farkas, J. B. Foresman, J. V. Ortiz, J. Cioslowski, D. J. Fox, Gaussian, Inc., Wallingford CT, (2013). [37] P.D. Johnson, N.V. Smith, Phys. Rev. B, 27 (1983) 2527-2530.

[38] F. Bussolotti, S. Kera, K. Kudo, A. Kahn, N. Ueno, Phys. Rev. Lett., 110 (2013) 267602.

[39] S. Duhm, G. Heimel, I. Salzmann, H. Glowatzki, R.L. Johnson, A. Vollmer, J.P. Rabe, N. Koch, Nature Mater., 7 (2008) 326-332.

[40] J. Meyer, M. Kroger, S. Hamwi, F. Gnam, T. Riedl, W. Kowalsky, A. Kahn, Appl. Phys. Lett., 96 (2010) 193302.

[41] Y. Wang, W.Y. Gao, S. Braun, W.R. Salaneck, F. Amy, C. Chan, A. Kahn, Appl. Phys. Lett., 87 (2005) 193501.

[42] I.G. Hill, A. Kahn, J. Appl. Phys., 86 (1999) 4515-4519.

[43] B.W. D'Andrade, S. Datta, S.R. Forrest, P. Djurovich, E. Polikarpov, M.E. Thompson, Org. Electron., 6 (2005) 11-20.

[44] J.D. Anderson, E.M. McDonald, P.A. Lee, M.L. Anderson, E.L. Ritchie, H.K. Hall, T. Hopkins, E.A. Mash, J. Wang, A. Padias, S. Thayumanavan, S. Barlow, S.R. Marder, G.E. Jabbour, S. Shaheen, B. Kippelen, N. Peyghambarian, R.M. Wightman, N.R. Armstrong, J. Am. Chem. Soc., 120 (1998) 9646-9655. [45] R.S. Ruoff, K.M. Kadish, P. Boulas, E.C.M. Chen, J. Phys. Chem., 99 (1995) 8843-8850. [46] E.S. Chen, E.C.M. Chen, N. Sane, L. Talley, N. Kozanecki, S. Shulze, J. Chem. Phys., 110 (1999) 9319-9329.

[47] H. Ishii, K. Sugiyama, E. Ito, K. Seki, Adv. Mater., 11 (1999) 605-625.

[48] J. Hwang, A. Wan, A. Kahn, Mater. Sci. Eng. R-Rep., 64 (2009) 1-31.

[49] G. Heimel, I. Salzmann, S. Duhm, N. Koch, Chem. Mat., 23 (2011) 359-377. 
A Graphical abstract
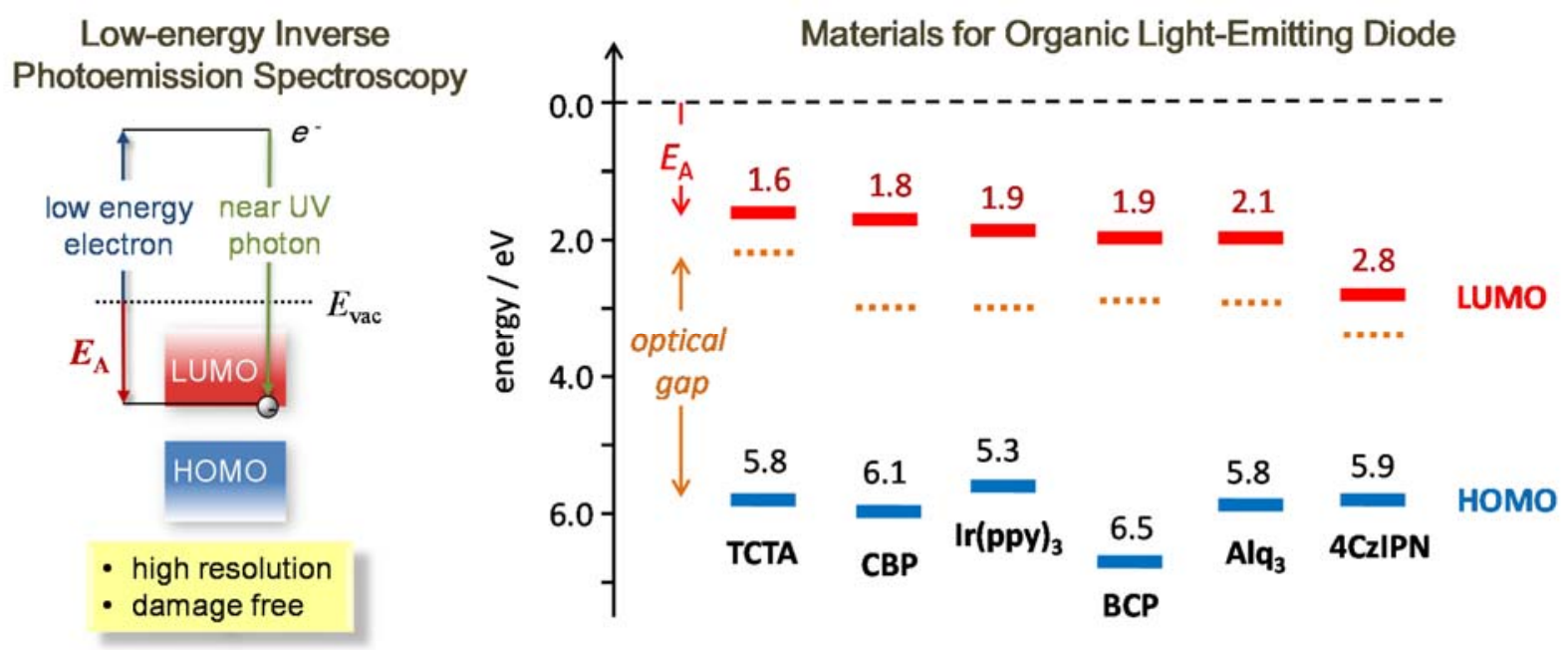